\title{
Semantic Conflict Mobilizes Self-Control in a Realistic Task
}

\author{
Tomasz Smolen $^{1}$ (D) $\cdot$ Adam Chuderski $^{2}$
}

Published online: 12 April 2017

(C) The Author(s) 2017. This article is an open access publication

\begin{abstract}
The study tested a hypothesis, based on the cognitive dissonance theory, that not only stimulus and response conflicts, as studied to date on the grounds of the influential conflict monitoring theory of human self-control, but also semantic incompatibility between complex cognitive representations, can mobilize such self-control. In two experiments, we applied a realistic task (simulated web feed), and manipulated the amount of semantic conflict (contradictory text messages), as well as the amount of tempting distractors (jokes and erotic pictures) that were supposed to be ignored. Experiment 1 demonstrated that semantic conflict mobilized self-control, as evidenced by participants better ignoring distraction. Experiment 2 showed that semantic conflict yielded the self-control depletion effect, analogous to the effects caused by a prolonged resistance to distraction, most probably because it mobilized self-control. The results extend the conflict monitoring theory, by implying that the detection of cognitive dissonance between incoming messages can also serve to regulate the strength of self-control.
\end{abstract}

Keywords Semantic conflict - Cognitive dissonance . Self-control $\cdot$ Motivation $\cdot$ Self-control depletion

Tomasz Smolen

tsmolen@up.krakow.pl

1 Psychology Department, Pedagogical University of Krakow, Podchorazych 2, 30-084, Krakow, Poland

2 Institute of Philosophy, Jagiellonian University in Krakow, Grodzka 52, 31-044, Krakow, Poland

\section{Introduction}

A crucial and, thus, intensively studied human mental faculty is self-control (also called executive control). Such control allows humans to initiate, regulate, coordinate, and suppress their thoughts and actions in a flexible and novel way, in order to reach adopted long-term goals, particularly in the face of conflicting stimulation and tempting but inadequate response tendencies. For instance, facing the choice between preparing for a boring but important exam and going to a pleasant but distracting party, most people will require mental effort and reflection to reject the party, and still some people fail to do so. The key role of effective self-control for coherent behavior can be seen when control has been disrupted (e.g., due to mental overload, tiredness, aging, neurological deficits, etc.), resulting in no longer being able to inhibit habitual but improper responses, prevent perseveration, overcome distraction, and organize actions into meaningful sequences (Banich 2009; Diamond 2013; Norman 1981; Shallice and Burgess 1993). Those deficits of control may lead to various personally and socially disruptive behaviors, such as poor scholastic achievement, procrastination, obesity, addictions, and violence (see Baumeister and Vohs 2004; Eigste et al. 2006; Malenka et al. 2009; Nigg et al. 2006).

However, even for people who control themselves quite effectively, strongly controlled, goal-focused processing seems to be energetically and cognitively costly, and often counter-effective (especially in highly skilled actions, when simple, automated strategies might be highly effective; Bargh et al. 2010). Moreover, strong control makes an agent focus exclusively on selected goals and actions, while other, temporarily ignored goals may become potentially more valuable but strong control may either prevent them from being attempted (Inzlicht et al. 2014) or make switching to them very difficult (Dang et al. 2014). As a result, for optimal 
behavior, an agent should strongly control her- or himself only when it is really necessary to correctly perform a task (usually novel, demanding, and important one), while exerting little self-control otherwise (the minimum control principle; Taatgen 2007). However, this results in a paradox: how can agents know when to apply strong but costly control, tightly focusing on goal-relevant processes, and when to withdraw them, relying on well-learned and thus less effortful behavioral schemata, without constantly "controlling" their own selfcontrol over behavior?

One solution to this paradox assumes that an agent monitors a simple global signal (simple enough not to require any complex processing), serving as a heuristic for deciding whether strong control is needed in a particular situation. Berlyne (1960) proposed that this signal may rely on response conflict present between potential actions, and strong conflict may drive organisms into particular actions. In their influential conflict monitoring theory, Botvinick et al. (2001) revived this approach, asking three interrelated questions: When should control start to regulate the response choice? How strongly should control regulate such a choice? When should control over the response choice be withdrawn? Botvinick et al. proposed that the control system (located at the anterior cingulate cortex; ACC) constantly monitors possible conflicts among potential responses, and resolves each conflict by enhancing the goal-relevant responses with the strength proportional to the conflict level. If the engagement of control eventually resolves the conflict, the strength of its intervention can be (gradually) decreased. Numerous studies supported predictions of the conflict monitoring theory (see Niendam et al. 2012). Although some other models have been proposed which assume that ACC, instead of responding to conflicts, learns by reinforcement errors (Holroyd and Coles 2002), risks (Brown and Braver 2007), or unexpected outcomes (Alexander and Brown 2011) that suggest a given situation requires high control (so that the required strength of control could be predicted in the future), the cross-model empirical tests (e.g., Yeung and Nieuwenhuis 2009) seem to support the conflict monitoring theory to a large extent. So, conflict monitoring remains a major theoretical account of how control is recruited and withdrawn, especially when it is supplemented with some specialized reinforcement learning mechanisms (see Chuderski and Smolen 2016; Verguts and Notebaert 2008).

However, there are two crucial issues that pertain to existing research supporting the conflict monitoring theory, which make it extremely difficult to generalize the predictions of this theory onto more complex and socially meaningful situations, beyond simple laboratory settings. Thus, it remains unclear whether, in more realistic, daily-life situations, the detection of conflicts indeed makes people control themselves more strongly.
First, virtually all of the tests of this theory were based on simplified stimulus-response tasks, such as the Stroop task and its variants (MacLeod 1991). For example, the flanker task requires responding to the direction of an arrow surrounded by other arrows pointing in the opposite direction. The color-word task consists of colored words that themselves name colors, and requires participants to run a less-learned process of naming colors, while suppressing a well-learned, and thus automatically activated, process of reading color names. The crucial finding in the Stroop paradigm, called the congruency effect, consists of increased response latency for incongruent stimuli (e.g., word "blue" presented in green), compared to congruent stimuli (e.g., "blue" in blue).

Indeed, numerous studies have shown that the congruency effect decreases when the imposed conflict level increases. For example, the effect decreases in sequences in which more incongruent stimuli are presented than congruent stimuli, in comparison to sequences that contain primarily congruent stimuli (Tzelgov et al. 1992). This observation was explained by Botvinick et al. (2001) as based on the mechanism of the conflict-driven mobilization of control (the increase in its strength), resulting from an overall higher level of conflict in the primarily incongruent sequences, compared to sequences that include more congruent than incongruent trials.

Although allowing strict experimental control, simplified tasks like Stroop have low ecological validity. In daily life, people do not need to name colors of colored words, or decide about directions of incompatible arrows. Furthermore, hundreds of trials of such tasks, commonly applied during an experiment, are extremely boring to participants, and the respective conflicts are pretty straightforward (e.g., the arrows point to either one or two directions). It is not certain whether people easily detect conflicts in a similar manner in more natural, complex settings, when many interesting events may divert their attention from the source of conflict (e.g., when interacting with emotionally or socially meaningful stimuli), and when the conflict is much harder to notice.

Second, but linked to the previous point, to date, virtually all studies related to the conflict monitoring theory pertained solely to conflicts among response tendencies, usually either simple vocalizations, such as saying a color/picture name, or manual actions, such as pressing a button or squeezing a dynamometer. However, since its very beginnings, psychological research has focused on conflicts beyond simple responding. For example, Kurt Lewin (1935) was one of the first scholars to investigate the conflicts between so called helping and hindering forces (e.g., personal and situational variables) that acted on a person, moving that person either toward or away from the adopted goal. That inspired Lewin's student, Leo Festinger (1957), to investigate conflicts between mental representations (in his terminology: cognitions), such as attitudes, opinions, or decisions, and to formalize the level of conflict between consonant (i.e., goal-relevant) and 
dissonant (i.e., goal-irrelevant) cognitions on the grounds of his seminal cognitive dissonance theory. According to Festinger, the level of conflict equals the weighted ratio of dissonances to the sum of all dissonances and consonances that are meaningful for a given situation (Festinger and Carlsmith 1959). Festinger predicted that motivation to counteract dissonance depends on the conflict level when expressed in this way. This prediction has been corroborated by Elliot and Devine (1994; for other studies supporting the cognitive dissonance theory see Cooper, 2007).

In the modern parlance of self-control research, such an increase in motivation can be referred to as the increased strength of self-control, or of executive control (Botvinick and Braver 2015). However, there were few studies that examined the influence of semantic conflict, which was understood as cognitive dissonance that resulted from the contradictory meaning of mental representations, on the strength of exerted control. It is clear that Stroop tasks, especially picture-word tasks (Glaser and Glaser 1982; La Heij and van den Hof 1995), yield some semantic conflict (e.g., a picture and a word mean different things), but (a) its nature is very simple in relation to that of cognitive dissonance (where two messages or beliefs mean the precisely opposite things), and (b) its behavioral effects can solely be explained in terms of memory retrieval (see Van Maanen et al. 2009) or response selection (see Chuderski and Smolen 2016). The latter possibility is indicated by the substantial response-set effects (i.e., a meaning not associated with a response yields minimal conflict; Proctor 1978). Two studies examined effects of more complex forms of semantic incompatibility. Van Veen et al. (2009) demonstrated that increased activation of the ACC accompanies the detection of cognitive dissonance between an attitude held by a participant and the contrasting argumentation provided, and predicts the respective attitude change. Kan et al. (2013) showed that presenting ambiguous sentences, whose initial interpretation might differ from the final (correct) interpretation, increases the strength of control (decreases response latency) in the subsequent incongruent trial of the color-word Stroop task. However, evidence showing that the incompatibility between the meaning of cognitive representations processed by an agent (possibly yielding cognitive dissonance) can affect the self-controlling behavior of that agent (e.g., can increase focus on task, or decrease the distractor proneness) is still insufficient.

Consequently, our aim was to examine predictions of the conflict monitoring theory (a) in a more "realistic" task than the laboratory tests applied to date (b) that would impose semantic conflicts comparable to cognitive dissonances, that is, when two mutually contradictory pieces of information had to be processed. In particular, we predicted that semantic conflicts introduced in our realistic task would be effectively detected by participants, and that these conflicts would mobilize self-control, as evidenced by the higher rate of picking target stimuli out of distractors when semantic conflict would be present, compared to when it would be absent.

\section{Experiment 1}

\section{Participants}

A total of 46 women and 36 men participated ( 82 people). They were recruited via adverts on social networking webpages. Mean age was 22.8 years $(S D=3.38$, range 18 38). Each participant received the equivalent of ten euros in local currency. Each person was informed that participation is voluntary, and that they can leave the lab at will. All participants had normal or corrected-to-normal vision.

\section{Experimental Design}

One challenge for an experimental study of self-control that aims to be carried out in a more natural setting is the design of a realistic task that, on one hand, can be applied using a computer, and requires relatively simple and well-measurable responses, whereas, on the other hand, still resembles activities that most people do on an everyday basis at their work or home. Our choice was a tool that requires both searching and reading short portions of information within a simplified web feed, in order to fulfill a task of gathering as much relevant knowledge on a given (realistic) problem as possible, and, then, answering one question regarding that problem. The task was difficult, as the target information was always accompanied by some distracting, task-irrelevant content. The two crucial manipulations in the task consisted of (a) increasing the amount of distracting information that accompanied the target information, and (b) introducing semantic incompatibility within the target information (some texts negated others).

Each participant dealt with four problems. In each problem, the screen was composed of a $3 \times 3$ matrix filled with textual or graphical portions of information (messages). The initial screen consisted of nine messages, one message per one matrix cell. Every $5 \mathrm{~s}$ (per cycle) one random message was substituted with another message. In total, 100 messages were presented in one problem (including the initial ones). The four problems were formulated as follows: "On the basis of the information provided, please...":

(a) judge the probability that IT company $\mathrm{X}$ will increase its headcount in the following year;

(b) describe how the human cortex works;

(c) explain how a computer processor works;

(d) evaluate what factors have the key role in supporting the existing political system in Ukraine. 
Importantly, in order to be maximally interesting for participants, the problems pertained to diverse topics. It was also important that the problems pertained to concrete objects or situations because, when more abstract problems where used in an analogous pilot study, the effects of semantic incompatibility were strongly decreased, probably because such incompatibility could not easily be detected.

Messages belonged to three categories. Target messages were short texts (but not shorter than 120 characters) providing information relevant for a problem to be solved. For instance, a target message could state that "Company X expects more sales next year and thus increases marketing costs...". However, some target messages were what we will call conflicting messages. Each such message was presented from two to five cycles after the corresponding target message, and contained a similar text to the latter message, except for the fact that its meaning was exactly opposite. For example, if the preceding target message stated that "Company $\mathrm{X}$ expects more sales next year and thus increases marketing costs...", then the conflicting message would say: "Company $\mathrm{X}$ expects less sales next year and will cut marketing costs...". If one message in such a pair contained negation, it was presented as either the first or second, at random.

Another category was distractor messages, which consisted of three subtypes. Fake messages (30\% of distractor messages) conveyed information superficially associated with a problem, but in fact irrelevant for it (e.g., "The employees of company X won the soccer cup in the 2013 sales departments competition..."). The remaining distractor messages (images) were either attractive graphics (30\%, funny cartoons and erotic images) or text jokes (40\%). Distractor messages were intended to capture participants' attention. We assumed that the high proportion of distractor messages within a particular problem should result in participants missing the target messages more often, compared to the low proportion. Since the target messages disappeared from the screen after $22.5 \mathrm{~s}$ on average (i.e., from 10 to $45 \mathrm{~s}$, depending on the number of cycles that each message was displayed for), the prolonged focus of attention on attractive, pleasant, but totally irrelevant distractor messages/images increased the chance that the incoming target message would disappear from the screen before attention was switched to that message.

The last category was noise messages, which conveyed either text information irrelevant for the problem, but in no way conflicting with the target messages (e.g., "Several national parks have been founded in Poland recently ..."), or images of supposedly non-distracting natural objects and landscapes. As the noise messages/images were rather dull and unattractive, we assumed that they would capture attention to a much lesser extent than would the distractor messages/images. The use of both the distractor and noise messages made the contents of the task relatively similar to internet portals, which usually contain a lot of irrelevant textual and graphical content that accompanies crucial information. The example screen of the task, including all types of messages, is presented in Fig. 1.

Both authors searched available web pages in order to collect erotic pictures, jokes, and noise images. A stimulus was included into the materials if both authors agreed that it fulfilled the criteria of a given category. In line with their strict instructions, the target, conflicting, and distracting messages were generated by a student, and were included into the materials only if both authors accepted them.

The task of each participant was to monitor and read messages that could be potentially informative with regard to the problem posed. Participants were instructed that they had to
Fig. 1 A demonstration of a screen in the realistic task used in Experiments 1 and 2, with particular types of messages that were presented in the task marked with arrows (note that no arrows were shown in the original screens). Texts represent English translations of the original messages (in the experiments, the task was administered in Polish)

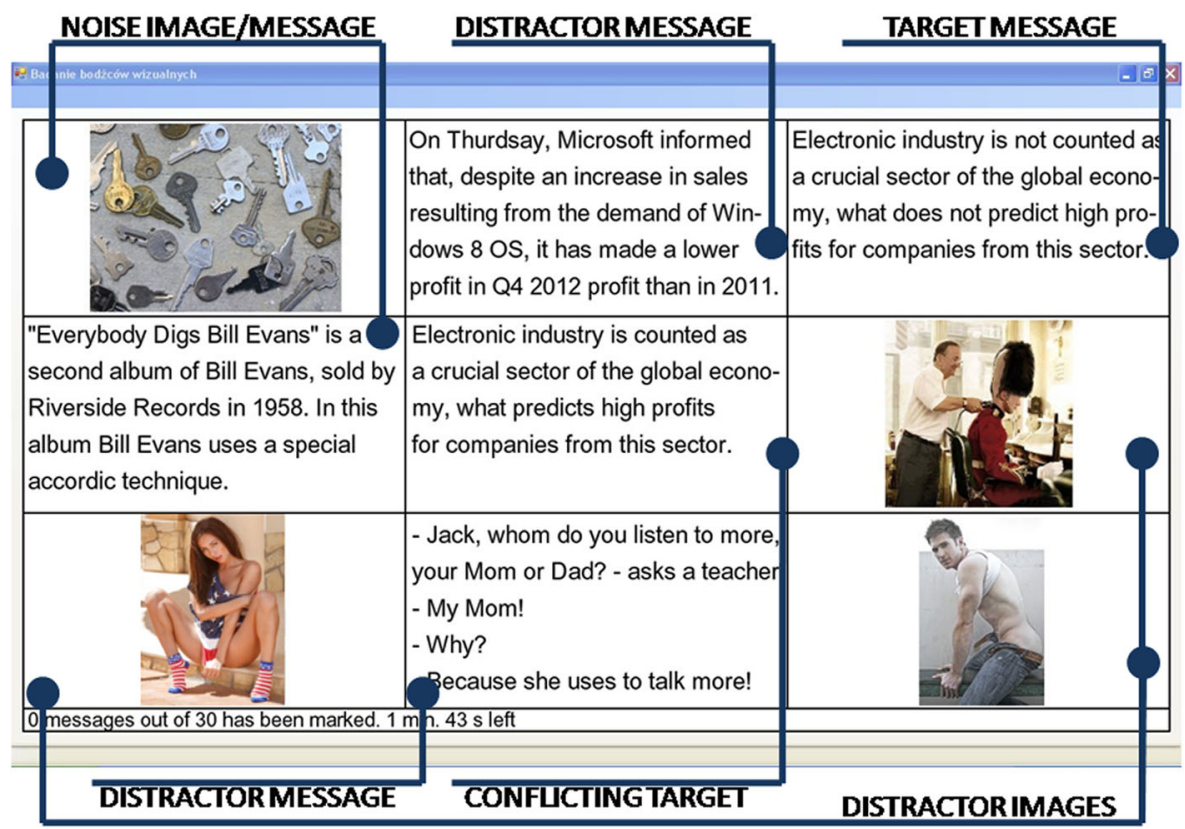


confirm that a certain message was a message conveying an important knowledge on the problem, via pressing the mouse button on that message. The clicked message was marked blue (regardless of whether it was a correct target message or an incorrect distractor/noise message, that is, there was no feedback on clicking). At the beginning of the experiment, in order to motivate participants to click on as many relevant messages as possible, people were informed that, after the sequence of messages, they would be provided with the messages they chose, which would help them to answer a question about a presented problem (e.g., "What is the probability that company X will increase its profits next year?"). Answering the question consisted of subjectively judging the respective probability. The participants were asked to provide as accurate judgements as possible, and they were not aware that their answers to the questions would not be evaluated after the study (in fact, as the problems were fictional, there could be no correct answers to the questions). However, as the instructions insisted on the most accurate performance on the message collection and further question answering, and the participants were told that they would be paid for their "accurate performance" (although eventually each participant was paid the same amount of money), we had good reasons to believe that our participants would be trying hard to focus on the task and mark as many target messages as possible (to ease their answers), thereby avoiding the distractors and noise.

Two crucial task parameters were the number of conflicting messages, and the number of distractor messages. In the noconflict condition, there were 30 target messages defined for a particular problem, but no conflicting messages were presented among them. In the conflict condition, out of 30 target messages, 10 were conflicting messages (i.e., they followed the target messages which had the meaning opposite to their own meaning). In the low-distraction condition, there were 10 distractor messages/images. In the high-distraction condition, as many as 60 distractor messages/images were presented. In order to obtain the 100-message/image sequences, in the former condition, 60 noise messages/images were used, while, in the latter, as few as 10 noise messages/images were included. For each participant and problem, the distractor and noise messages/images were sampled randomly without replacement, using a pool of about 1200 distractors and 1500 noise messages/images. The number of stimuli of each type for each condition of the task is summarized in Table 1.

The independent variables were semantic conflict (either present in 10 conflicting messages, or absent when no conflicting messages were used), and distraction (either low when 10 distractor messages/images were shown, or high when 60 distractor messages/images were displayed). For each participant, we applied one problem in each of four possible conditions (no-conflict/low-distraction, no-conflict/high-distraction, conflict/low-distraction, and conflict/high-distraction), resulting in a $2 \times 2$ "within-subjects" design. Particular problems and conditions were assigned randomly for each participant.

We expected that high distraction would decrease performance accuracy (i.e., people would be looking more frequently at fake messages, erotic pictures, and jokes, instead of selecting the target messages). Crucially, we also expected that the distraction effect (i.e., the positive error rate difference between the high-distraction and the low-distraction condition) would be present in the no-conflict condition, but would be attenuated by increased control in the conflict condition. We assumed that people, after detecting semantic conflicts (i.e., conflicting messages), which were expected to act as a kind of red alarm, would more effectively ignore distractors and focus on the task (i.e., they would detect and read target messages more carefully). Such an effect might indicate that the control system could be mobilized by increased semantic conflict, and, as a result, distraction could be more effectively rejected.

\section{Procedure and Scoring}

Groups of several people were tested using the above mentioned task for roughly one hour. Testing took place in a large, dimly lit room. Standard PC workstations were used. Each participant occupied a visually isolated desk, and was asked to adopt the most comfortable sitting position.
Table 1 Number of stimuli of each type in each condition of Experiment 1

\begin{tabular}{llllll}
\hline & \multicolumn{2}{l}{ Condition } & & & \\
\cline { 2 - 3 } & No conflict & & Conflict & \\
\hline Messages & Low distraction & High distraction & & Low distraction & High distraction \\
\hline Target messages & 30 & 30 & 20 & 20 \\
Conflicting messages & 0 & 0 & 10 & 10 \\
Distractor messages/images & 10 & 60 & 10 & 60 \\
Noise messages/images & 60 & 10 & 60 & 10 \\
Total number of messages/images & 100 & 100 & 100 & 100 \\
\hline
\end{tabular}


The dependent variable (DV) approximated $d$ ' statistics of the signal detection theory, and was calculated, for each problem, as the proportion of missed target messages (i.e., reversed rate of hits; ERR), increased by the weighted proportion of incorrectly marked distractor and noise messages/images (i.e., the rate of false alarms, FA). The conflicting messages were excluded from the DV calculation, as they were only intended to elicit conflict, and because they were more often missed $(M=.46)$ than were the target messages $(M=.31)$. The weight used reflected the ratio of target messages (either 20 or 30 , in the conflict or the no-conflict condition, respectively) to the sum of distractor and noise messages (70). Specifically, DV equaled ERR +0.286 FA in the conflict condition, and ERR +0.429 FA in the no-conflict condition. This correction of participants' decisional bias was meant to account for the individual response tendencies, as people who generally tended to respond more often (i.e., had lower response threshold and thus more frequently responded on random) also had a larger chance of hitting the target (see Snodgrass and Corwin 1988). The specific weight indicated the probability of a participant marking the target message by chance when clicking messages at random, as indicated by their FA. In the remainder of the text, we refer to our corrected error rate simply as error rate. Participants only received the overall accuracy feedback at the end of each problem, by obtaining information on the number of correctly marked target messages (which were listed, in order to ease the answer to the question). Since, due to the multichannel form of information presentation, we were not able to control the moment at which participants started attending to a given message/image, we did not analyze response times.

We tested the influence of our two factors (distraction: high or low, and conflict: present or absent), as well as their interactive effect on DV, in one statistical model submitted to ANOVA.

\section{Results and Discussion}

The mean error rate was $.33(S D=.11)$. It ranged from $M=.15$ to $M=.64$ for particular participants, suggesting that they followed instructions for the task. The mean error rates and their $95 \%$ confidence intervals for all experimental conditions are presented in Fig. 2.

Both factors yielded significant main effects in ANOVA. First, in the high-distraction condition, the error rate was higher $(M=.39)$ than in the low-distraction condition $(M=.28), F(1,81)=55.59, p<.001, \eta^{2}=.41$. This fact implied that fake messages, erotic images, and funny cartoons, originally aimed at capturing people's attention, indeed diverted participants from fulfilling the task, and constituted a substantial source of distraction for the control system. Second, there was no significant difference in error rate between the conflict and no-conflict conditions, $F(1,81)=0.12$,

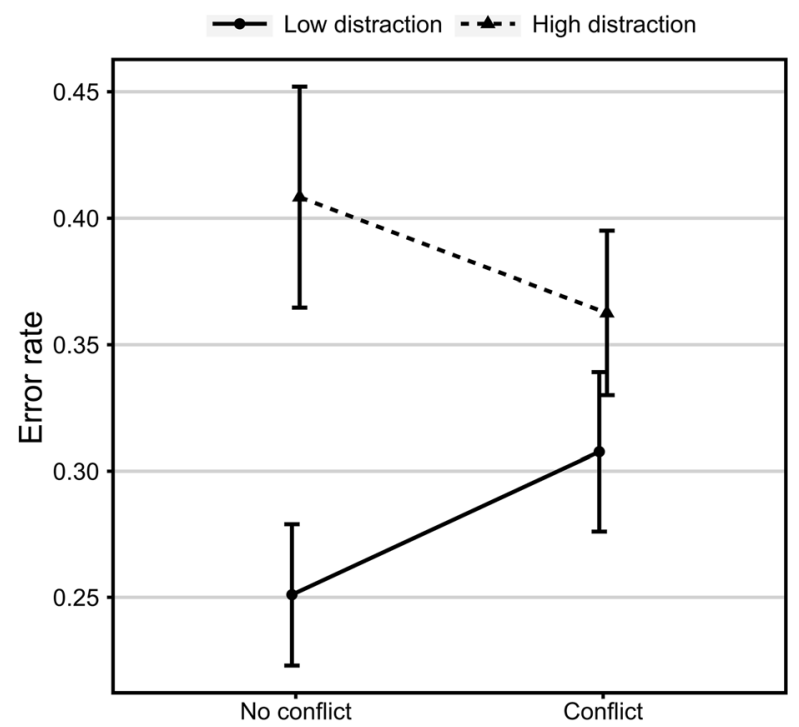

Fig. 2 Mean rate of target message omission, corrected for false alarms (i.e., error rate), in the conflict versus the no-conflict condition, for the low- (dashed line) versus the high-distraction (solid line) condition. Bars represent $95 \%$ confidence intervals

meaning that conflict did not affect the accuracy of the target messages detection per se.

The most important effect was the two-way interaction of investigated factors (see Fig. 2), which was significant $F(1$, $81)=15.24, p<.001$, and substantial, $\eta^{2}=.94$. Tukey's HSD test showed that there was a significant difference between the high- and the low-distraction condition both in the no-conflict $(p<.001)$ and the conflict $(p=.008)$ condition, however, the conflict condition yielded a substantially smaller distraction effect $(\Delta M=.05)$ than the no-conflict condition $(\Delta M=.16)$. Thus, it can be concluded that, in concord with our hypothesis, the introduction of semantic conflicts most likely seemed to mobilize control over distractors.

\section{Experiment 2}

Experiment 2 aimed to further validate the conclusion reached in Experiment 1, by observing the potential effects that the supposedly mobilized self-control (due to semantic conflicts) can have on the human ability to exert further control. Our examination was rooted in numerous studies which suggested that, after prolonged recruitment of control (like ignoring intrusive thoughts, temptations, and distractors), the ability to exert self-control becomes temporarily depleted (Muraven and Baumeister 2000; Muraven et al. 1998; for a comprehensive review see Hagger et al. 2010). Although the evidence for such self-control depletion have recently been questioned (Carter et al. 2015; Carter and McCullough 2014; Xu et al. 2014), other studies proposed that selfcontrol may indeed become released with elapsing time, due to shifts in motivation (Inzlicht et al. 2014), or because of 
drops in the subjective value of self-control, while the pleasure/leisure value increases (Kool and Botvinick 2014; Shenhav et al. 2014). Thus, given that strong self-control in one task often yields some depleting effect on performance in another task (Hagger et al. 2010), regardless of the underlying mechanisms, in Experiment 2, we expected that the introduction of substantial semantic conflict into our realistic task would decrease participants' performance on a subsequent attention control test (the antisaccade task), compared to a control group, in which participants would face negligible semantic conflict. This would be another way to demonstrate that semantic conflicts can mobilize the control system - via depletion/demotivation effects which can result from such a mobilization.

\section{Participants}

A total of 110 women and 55 men participated (165 people). Eight participants were removed from the antisaccade task data analysis due to their below chance level performance on the task. In total, $4.8 \%$ of the sample was discarded due to the floor effect. The recruitment and gratification were the same as in Experiment 1. Mean age was 23.28 years $(S D=4.79$, range 18-46). The participants were randomly assigned to one of three study groups in the order in which they appeared in the experiment: the control group $(N=46)$, the high-conflict group $(N=51)$, and the high-distraction group $(N=60)$.

\section{Experimental Design and Procedure}

We used the same realistic task as in Experiment 1, with the following modifications. First, in order to make sure that the results would not depend on the four selected problems, the pool of problems now included as many as 16 problems (which, like in Experiment 1, each included 100 messages). We used the four problems from Experiment 1, plus the twelve listed below:

1. explain how the human brain/vision/body or computer/ drainage/Krakow drainage system works

2. analyze a new investment of IT company $\mathrm{X}$ in a mobile phone system;

3. evaluate the prospects of IT company X in light of the new investment in a mobile phone system;
4. evaluate financial prospects of the IT company $\mathrm{X}$ in the coming year;

5. evaluate what factors have the most important role in determining the EU policy towards Ukraine;

6. evaluate the EU policy towards Moldova;

7. approximate the chances that the Moldovan political system will change in the near future.

Second, there were three variants of the task depending on group. The control group was presented with four problems randomly drawn from the pool of problems. As in Experiment 1 , two problems included a low number (10) of distractor messages/images (and 60 noise messages/images), and two, a high number (60) of distractor messages/images (and 10 noise messages/images). As in Experiment 1, the difference in error rate between the high- and low-distraction conditions indexed the distraction effect. Each problem also included 26 target messages, and 4 conflicting messages. The latter constituted very low semantic conflict. The high-distraction group received a task variant with twice as many problems as did the control group (i.e., four in the low- and four in the highdistraction condition). This meant that twice as many to-berejected distractors were presented to this group, compared to the control group. In order to equate the total number (16) of conflicting messages in the high-distraction and the control group, each problem in the former group contained only two such messages (as well as 28 target messages). All other task parameters were the same as in the control group. Crucially, in the high-conflict group, the task variant with four problems was applied, however, each problem included as many as 10 conflicting messages (and 20 target messages). That was an increase of semantic conflict by $150 \%$ in comparison to the two remaining groups. All other task parameters were the same as in the control group. The numbers of problems and the numbers of each type of message per problem are presented in Table 2. DV for the realistic task was calculated identically as in Experiment 1.

The self-control depletion effect was quantified by a wellestablished attention control test, the antisaccade task (Hallett 1978). The task requires making fast eye movements away from a distractor. We applied the task just before (pretest) and just after (posttest) the realistic task. Each task administration consisted of 40 self-paced trials. Each trial consisted of four events. First, a cue was presented for $1.5 \mathrm{~s}$ to prompt
Table 2 Number and type of problems in each of groups in Experiment 2

\begin{tabular}{|c|c|c|c|c|c|}
\hline \multirow[b]{2}{*}{ Group } & \multirow{2}{*}{$\begin{array}{l}\text { Number of } \\
\text { problems }\end{array}$} & \multicolumn{2}{|c|}{ Number of distractor messages/images } & \multicolumn{2}{|c|}{ Number of conflicting messages } \\
\hline & & On average per problem & Total & Per problem & Total \\
\hline Control & 4 & 35 & 140 & 4 & 16 \\
\hline High-distraction & 8 & 35 & 280 & 2 & 16 \\
\hline High-conflict & 4 & 35 & 140 & 10 & 40 \\
\hline
\end{tabular}


subjects to look at the side opposite to a rapidly flashing black square. Next, a fixation point was presented in the center of the screen for 1-2 $\mathrm{s}$. Then, the flashing square was shown in the middle of the left or right side of the screen, about $16 \mathrm{~cm}$ from the fixation point, for $0.15 \mathrm{~s}$. Finally, a small dark gray letter (in pretest) or arrow (in posttest) was presented in the middle of the opposite side of the screen to the square for only $0.2 \mathrm{~s}$ before being replaced by a mask. The task was to look away from the flashing square, in order to detect the direction of the arrow or the identity of the letter, and to press the associated key. The index of self-control depletion was the error rate in the post-test minus the error rate in the pre-test. Although we note that, to date, the antisaccade task has not been used in depletion studies, it is a very reliable test validly tapping the strength of executive control, which is commonly used in cognitive psychology (Unsworth et al. 2011) and in psychometrics (Shipstead et al. 2014). So, any control depletion effects should be detected by means of this task.

Our hypotheses were the following. First, in the realistic task we expected a higher error rate in the high-distraction condition (i.e., that with 60 distractor messages/images) than in the low-distraction condition (that with 10 distractor messages/images), matching the results of Experiment 1. This effect would indicate that increased distraction also diverted participants' attention from the target messages in Experiment 2. Second, we assumed that the load on self-control imposed by the realistic task should be relatively low in the control group. In contrast, in the high-distraction group, as the task duration was doubled, and thus it included twice as much distractors, we expected a larger recruitment of control to be necessary, and, thus, we predicted a significantly larger depletion index, in comparison to the control group. Most importantly, we expected that a presumably larger load on selfcontrol in the high-conflict group would also result in a larger depletion index, compared to the control group. As these two latter groups differed only in the number of conflicting messages, a larger depletion effect found in the high-conflict group could only be assigned to the increased semantic conflict. The latter was our main prediction for Experiment 2. We had no specific hypothesis on the relation of the depletion index between the high-distraction and the high-conflict group.

We note that the "high-distraction" group differed from the control and the high-conflict group not only by the doubled number of distractor messages/images, but also by the doubled duration, thus a larger load on controlled performance in this group could not be associated solely with the former factor. However, what was relevant about this group for the current study was just a larger load in general (arising either due to more episodes of distraction inhibition or more time for control to be active, or both). In short, this group served only as a baseline depletion group, in which a "standard" depletion effect was expected to occur. Such an effect would validate our procedure as being comparable to existing studies which induced depletion effects by imposing larger distraction to be resisted/rejected (see Hagger et al. 2010).

\section{Results and Discussion}

The error rates for the pretest, the realistic task, and the posttest, for the respective groups, are presented in Table 3. First, we submitted error rate in the realistic task to ANOVA, with three levels of the group factor (the control, high-distraction, and high-control groups) and two levels of the distraction factor (low vs. high). The main effect of the latter factor was highly significant, $F(1,324)=84.6, p<.001, \eta^{2}=.20$, indicating an increase in error rate of $\Delta M=.13$ from the low- to the high-distraction condition. The group factor also yielded a significant difference, $F(2,324)=9.15, p<.001, \eta^{2}=.04$, indicating that error rate in the high-conflict group was higher by $\Delta M=.07$ compared to the high-distraction group, $F(1$, $324)=18.25, p<.001$. There was no significant interactive effect of the group and the distraction, $p=.41$. Although this latter result of Experiment 2 does not match the finding of Experiment 1, the discrepancy between both experiments is difficult to interpret, because both experiments differed in design (between- vs. within-subjects, respectively).

Most importantly, we submitted the posttest error rate in the antisaccade task to ANCOVA, again with three groups as the factor, and the pretest error rate as the covariate (the latter in order to account for a visible difference in the pretest performance between the groups). There was a significantly larger depletion effect for both the high-distraction and the highcontrol group, in comparison to the control group, $F(2$, $153)=3.70, p=.027, \eta^{2}=.02$. Error rate in the posttest, corrected by using the pretest error rate, was higher by $\Delta M=.044$ in the high-conflict group, $F(1,153)=3.06$, $p=.041$, and by $\Delta M=.063$ in the high distraction group, $F(1,153)=4.34, p=.019$ (both contrasts one-tailed, as directional hypotheses assuming larger depletion were tested), in comparison to the control group. As suggested by a reviewer, we ran alternative analysis with difference between post-test and pre-test in the antisaccade task as dependent variable, and the error rate in the realistic task as the covariate. Both the

Table 3 Mean error rate (and $S D$ ) in the pre-test, realistic task, and post-test in Experiment 2

\begin{tabular}{llll}
\hline Group: & Control & High-distraction & High-conflict \\
\hline Antisaccade pre-test & $.18(.18)$ & $.19(.15)$ & $.14(.15)$ \\
Low-distraction condition & $.28(.13)$ & $.27(.10)$ & $.32(.14)$ \\
High-distraction condition & $.42(.13)$ & $.37(.13)$ & $.46(.12)$ \\
Antisaccade post-test & $.14(.14)$ & $.21(.21)$ & $.16(.15)$ \\
\hline
\end{tabular}

$N=165$ for the realistic task. $N=157$ for the antisaccade task 
main effect of group factor, $F(2,153)=3.36, p=.037$, $\eta^{2}=0.42$, and the respective contrasts for the high-conflict, $F(1,153), p=.032$, and the high-conflict distraction group, $F(1,153), p=.036$ (both one-tailed), matched the results of the preceding ANCOVA. Given that the average error rate in the antisaccade task was $M=.182$, the relative depletion effect for both the high-conflict and the high-distraction group amounted a substantial value of the quarter of total errors. However, as indicated by values of Cohen's $d=.42$ and $d=.48$, respectively, the observed strength of the depletion effect was only moderate, and in line with the estimates provided by Carter and McCullough (2014). Figure 3 presents the relative change in the antisaccade error rate between the preand posttest, for consecutive groups.

\section{Conclusion}

We tested the hypothesis that increased perception of semantic conflict affects the exerted strength of self-control. Using a novel, realistic task that resembled web feeds commonly used by people at home and work, Experiment 1 showed that introducing semantic conflict, evoked by placing the mutually contradictory messages within the stream of information shown to participants, mobilized their self-control, allowing them to deal more effectively with distractors. Experiment 2 verified, with the same task, that a permanently increased level of semantic conflict (most probably resulting in permanently increased control strength) yielded comparable depletion

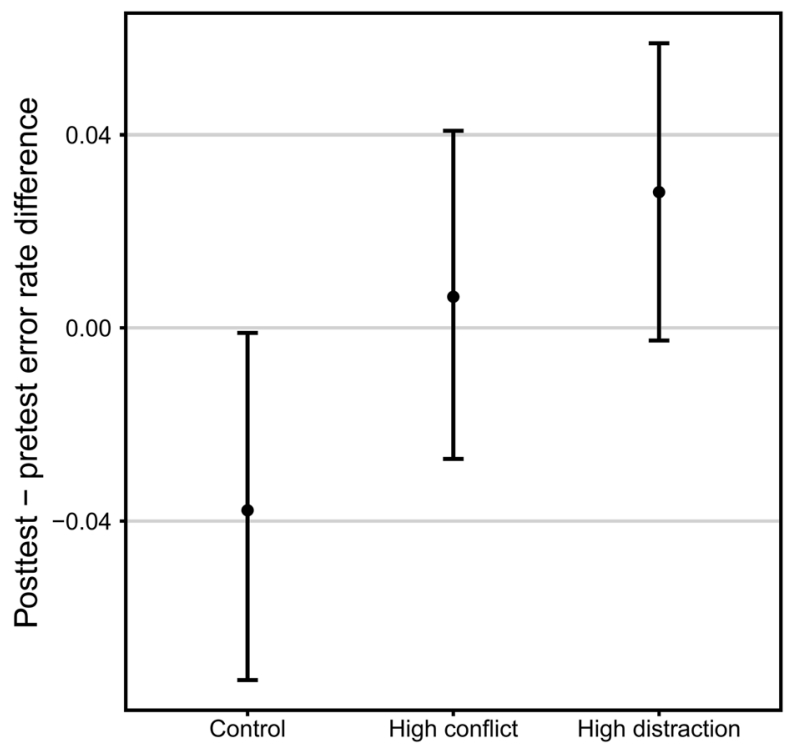

Fig. 3 Mean change in the error rate from the pretest to the posttest of the antisaccade task, for the control, high-distraction, and high-conflict group. Bars represent $95 \%$ confidence intervals. Note that because of some practice effect present in the control group (the drop in error rate in the post-test by .03), the two experimental groups should be compared to the control group, not to the zero line effects as did the need to reject tempting distractors. Thus, both experiments confirmed the hypothesis posed.

The present study suggests that the predictions of the conflict monitoring theory, originally pertaining to response conflicts, can be generalized beyond their original realm, onto more complex and higher-level conflicts pertaining to processing of semantically incompatible messages, as (indirectly) predicted by the cognitive dissonance theory proposed by Festinger (1957). This finding extends the central assumption of the conflict monitoring theory, which claims that the brain constantly evaluates conflicts within the information stream, and regulates the self-control strength accordingly. As such, our data seem to strengthen the psychological plausibility of this theory, showing that it predicts human selfcontrolling behavior not only in laboratories, but also in everyday, socially meaningful situations.

Overall, the study demonstrated that the crucial effect of cognitive dissonance on motivation, to date supported by the myriad of studies originating from the Festinger theory (see Cooper 2007), can also be applied to the much more recent theorizing on self-control. It seems that the intensity of both motivation and self-control is similarly affected by the semantic conflict between cognitive representations. Such conflict may act as a red alarm, informing the human mind that something is going wrong with its information processing and/or its environment, and that more careful, as well as task-focused, behavior is required. This fact also suggests, in line with recent proposals (e.g., Botvinick and Braver 2015; Inzlicht et al. 2014), that self-control and motivation may be more strongly intertwined than it was supposed earlier. However the nature of their interaction remains to be discovered in future research.

Acknowledgements We are grateful to J. Wójcik for designing the problems, J. Sadowsky for programming the realistic task, and K. Cipora for conducting the experiments. Experiment 1 was presented during EuroAsianPacific Joint Conference on Cognitive Science, Torino, 2015.

\section{Compliance with Ethical Standards}

Funding This work was funded by the National Science Centre of Poland (grant number 2011/01/D/HS6/00467).

Ethical Approval All procedures performed in studies involving human participants were in accordance with the ethical standards of the institutional and/or national research committee and with the 1964 Helsinki declaration and its later amendments or comparable ethical standards. This article does not contain any studies with animals performed by any of the authors.

Informed Consent Informed consent was obtained from all individual participants included in the study.

Conflict of Interest Tomasz Smolen declares that he has no conflict of interest. Adam Chuderski declares that he has no conflict of interest. 
Open Access This article is distributed under the terms of the Creative Commons Attribution 4.0 International License (http:// creativecommons.org/licenses/by/4.0/), which permits unrestricted use, distribution, and reproduction in any medium, provided you give appropriate credit to the original author(s) and the source, provide a link to the Creative Commons license, and indicate if changes were made.

\section{References}

Alexander, W. H., \& Brown, J. W. (2011). Medial prefrontal cortex as an action-outcome predictor. Nature Neuroscience, 14, 1338-1344. doi:10.1038/nn.2921.

Banich, M. T. (2009). Executive function: The search for an integrated account. Current Directions in Psychological Science, 18(2), 89-94. doi:10.1111/j.1467-8721.2009.01615.x.

Bargh, J. A., Gollwitzer, P. M., \& Oettingen, G. (2010). Motivation. In S. Fiske, D. Gilbert, \& G. Lindzey (Eds.), Handbook of social psychology (pp. 268-316). New York: Wiley.

Baumeister, R. F., \& Vohs, K. D. (Eds.). (2004). Handbook of self-regulation: Research, theory, and applications. New York: Guilford.

Berlyne, D. E. (1960). Conflict, arousal, and curiosity. New York: McGraw-Hill.

Botvinick, M. M., \& Braver, T. S. (2015). Motivation and cognitive control: From behavior to neural mechanism. Annual Review of Psychology, 66, 83-113. doi:10.1146/annurev-psych-010814-015044.

Botvinick, M. M., Braver, T. S., Barch, D. M., Carter, C. S., \& Cohen, J. D. (2001). Conflict monitoring and cognitive control. Psychological Review, 108, 624-652. doi:10.1037/0033-295X.108.3.624.

Brown, J. W., \& Braver, T. S. (2007). Risk prediction and aversion by anterior cingulate cortex. Cognitive, Affective, \& Behavioral Neuroscience, 7, 266-277. doi:10.3758/CABN.7.4.266.

Carter, E. C., \& McCullough, M. E. (2014). Publication bias and the limited strength model of self-control: Has the evidence for ego depletion been overestimated? Frontiers in Psychology, 5, 823. doi:10.3389/fpsyg.2014.00823.

Carter, E. C., Kofler, L. M., Forster, D. E., \& McCollough, M. E. (2015). A series of meta-analytic tests of the depletion effect: Self-control does not seem to rely on a limited resource. Journal of Experimental Psychology: General, 144, 796-815. doi:10.1037/xge0000083.

Chuderski, A., \& Smolen, T. (2016). An integrated model of utility-based evaluation and resolution of conflicts in the Stroop task. Psychological Review, 123, 255-290. doi:10.1037/a0039979.

Cooper, J. (2007). Cognitive dissonance: 50 years of a classic theory. Los Angeles: Sage.

Dang, J., Xiao, S., \& Dewitte, S. (2014). Self-control depletion is more than motivational switch from work to fun: The indispensable role of cognitive adaptation. Frontiers in Psychology, 5, 933. doi:10.3389/fpsyg.2014.00933.

Diamond, A. (2013). Executive functions. Annual Review of Psychology, 64, 135-168. doi:10.1146/annurev-psych-113011-143750.

Eigste, I.-M., Zayas, V., Mischel, W., Shoda, Y., Ayduk, O., Dadlani, M. B., Davidson, M. C., Aber, J. L., \& Casey, B. J. (2006). Predicting cognitive control from preschool to late adolescence and young adulthood. Psychological Science, 17(6), 478-484. doi:10.1111/j.1467-9280.2006.01732.x.

Elliot, A. J., \& Devine, P. G. (1994). On the motivational nature of cognitive dissonance: Dissonance as psychological discomfort. Journal of Personality and Social Psychology, 67, 382-394. doi:10.1037/0022-3514.67.3.382.

Festinger, L. (1957). A theory of cognitive dissonance. Stanford: Stanford University Press.
Festinger, L., \& Carlsmith, J. M. (1959). Cognitive consequences of forced compliance. Journal of Abnormal and Social Psychology, 58, 203-210. doi:10.1037/h0041593.

Glaser, M. O., \& Glaser, W. R. (1982). Time course analysis of Stroop phenomenon. Journal of Experimental Psychology: Human Perception and Performance, 8, 875-894.

Hagger, M. S., Wood, C., Stiff, C., \& Chatzisarantis, N. L. D. (2010). Ego depletion and the strength model of self-control: a meta-analysis. Psychological Bulletin, 136, 495-525. doi:10.1037/a0019486.

Hallett, P. E. (1978). Primary and secondary saccades to goals defined by instructions. Vision Research, 18, 1279-1296.

Holroyd, C. B., \& Coles, M. G. H. (2002). The neural basis of human error processing: Reinforcement learning, dopamine, and the errorrelated negativity. Psychological Review, 109, 679-709.

Inzlicht, M., Schmeichel, B. J., \& Macrae, C. N. (2014). Why self-control seems (but may be not) limited. Trends in Cognitive Sciences, 18, 127-133. doi:10.1016/j.tics.2013.12.009.

Kan, I. P., Teubner-Rhodes, S., Drummey, A. B., Nutile, L., Krupa, L., \& Novick, J. M. (2013). To adapt or not to adapt: The question of domain-general cognitive control. Cognition, 129, 637-651. doi: 10.1016/j.cognition.2013.09.001.

Kool, W., \& Botvinick, M. (2014). A labor/leisure tradeoff in cognitive control. Journal of Experimental Psychology: General, 143, 131141. doi: $10.1037 / \mathrm{a} 0031048$

La Heij, W., \& van den Hof, E. (1995). Picture-word interference increases with target-set size. Psychological Research, 58, 199-133.

Lewin, K. (1935). A dynamic theory of Personality. New York: McGraw-Hill.

MacLeod, C. M. (1991). Half a century of a research on the Stroop effects: an integrative review. Psychological Bulletin, 109, 163-203.

Malenka, R. C., Nestler, E. J., \& Hyman, S. E. (2009). Chapter 13: Higher cognitive function and behavioral control. In A. Sydor \& R. Y. Brown (Eds.), Molecular neuropharmacology: A Foundation for Clinical Neuroscience (pp. 313-321). New York: McGraw-Hill Medical.

Muraven, M. R., \& Baumeister, R. F. (2000). Self-regulation and depletion of limited resources: Does self-control resembles a muscle? Psychological Bulletin, 126, 247-259.

Muraven, M. R., Tice, D. M., \& Baumeister, R. F. (1998). Self-control as a limited resource: Regulatory depletion patterns. Journal of Personality and Social Psychology, 74, 774-789.

Niendam, T. A., Laird, A. R., Ray, K. L., Dean, Y. M., Glahn, D. C., \& Carter, C. S. (2012). Metaanalytic evidence for a superordinate cognitive control network subserving diverse executive functions. Cognitive, Affective, \& Behavioral Neuroscience, 12, 241-268. doi:10.3758/s13415-011-0083-5.

Nigg, J. T., Wong, M. M., Martel, M., Jester, J. M., Puttler, L. I., Glass, J. M., Adams, K. M., Fitzgerald, H. E., \& Zucker, R. A. (2006). Poor response inhibition as a predictor of problem drinking and illicit drug use in adolescents at risk for alcoholism and other substance use disorders. Journal of the American Academy of Child and Adolescent Psychiatry, 45, 468-475. doi:10.1097/01.chi.0000199028.76452.a9.

Norman, D. A. (1981). Categorization of action slips. Psychological Review, 88, 1-15.

Proctor, R. W. (1978). Sources of color-word interference in the Stroop color-naming task. Perception \& Psychophysics, 23, 413-419.

Shallice, T., \& Burgess, P. W. (1993). Supervisory control of action and thought selection. In A. Baddeley \& L. Weiskrantz (Eds.), Attention: Selection, awareness, and control (pp. 171-181). Oxford: Clarendon Press.

Shenhav, A., Botvinick, M. M., \& Cohen, J. D. (2014). The expected value of control: An integrative theory of anterior cingulate cortex function. Neuron, 79, 217-240. doi:10.1016/j.neuron.2013.07.007.

Shipstead, Z., Lindsey, D. R. B., Marshall, R. L., \& Engle, R. W. (2014). The mechanisms of working memory capacity: Primary memory, 
secondary memory, and attention control. Journal of Memory and Language, 72, 116-141. doi:10.1016/j.jml.2014.01.004.

Snodgrass, J. G., \& Corwin, J. (1988). Pragmatics of measuring recognition memory: Applications to dementia and amnesia. Journal of Experimental Psychology: General, 117, 34-50.

Taatgen, N. A. (2007). The minimal control principle. In W. Gray (Ed.), Integrated models of cognitive systems (pp. 368-379). New York: Oxford University Press.

Tzelgov, J., Henik, A., \& Berger, J. (1992). Controlling Stroop effects by manipulating expectations for color words. Memory and Cognition, 20, 727-735.

Unsworth, N., Spillers, G. J., Brewer, G. A., \& McMillan, B. D. (2011). Attention control and the antisaccade task: a response time distribution analysis. Acta Psychologica, 137, 90-100. doi:10.1016/j.actpsy.2011.03.004.

Van Maanen, L., Van Rijn, H., \& Borst, J. P. (2009). Stroop and pictureword interference are two sides of the same coin. Psychonomic Bulletin \& Review, 16, 987-999. doi:10.3758/PBR.16.6.987.
Van Veen, V., Krug, M. K., Schooler, J. W., \& Carter, C. S. (2009). Neural activity predicts attitude change in cognitive dissonance. Nature Neuroscience, 12, 1469-1474. doi:10.1038/nn.2413.

Verguts, T., \& Notebaert, W. (2008). Hebbian learning of cognitive control. Psychological Review, 115, 518-525. doi:10.1037/0033-295X.115.2.518.

Xu, X., Demos, K. E., Leahey, T. M., Hart, C. N., Trautvetter, J., Coward, P., Middleton, K. R., \& Wing, R. R. (2014). Failure to replicate depletion of self-control. PloS One, 9(10), e109950. doi:10.1371/journal.pone.0109950.

Yeung, N., \& Nieuwenhuis, S. (2009). Dissociating response conflict and error likelihood in anterior cingulate cortex. Journal of Neuroscience, 29, 14506-14510. doi:10.1523/JNEUROSCI.3615-09.2009. 